\title{
Drifting Behaviour of Aquatic Mites and Regulating Ecological Parameters in Khankragad Stream, A Springfed Trubutary of Alaknanda River, Rudrarayag, Garhwal, Uttarakhand, India
}

\author{
Shailza Negi ${ }^{1}$ Pankaj Bahuguna ${ }^{2 *}$ Anoop Dobriyal ${ }^{1}$ \\ ${ }^{1}$ Department of Zoology, HNB Garhwal University (A Central University), BGR Campus, Pauri (Garhwal) -246001 \\ Uttarakhand, India \\ ${ }^{2}$ Department of Zoology, BD Govt. P.G. College Lansdowne, Jaiharikhal-246193, Pauri Garhwal, Uttarakhand, \\ India.
}

*Corresponding Author Email id: pankajpaurii@gmail.com

Received: 17.04.2021; Revised: 11.05.2021; Accepted: 12.06.2021

OSociety for Himalayan Action Research and Development

\begin{abstract}
Water mites are a large group of macroinvertebrates that are very vulnerable to contamination and environmental changes. As a result, they are often used to monitor water quality. Khankra gad stream, a third-order perennial spring-fed stream of the Alaknanda River in Uttarakhand, India, was studied to determine the drift behavior of water mites and water quality. Drift nets were set up for 24 hours in both spots of the Khankra stream where there was little or no human influence over a two-year sampling period (June 2018-20). Every four hours, the nets were changed out with new ones. Water mites signify a particular drift month and diel drift pattern. The majority of the drift mite species were present in considerably higher numbers in the daytime. During the two-year study period, a total of 2503 mite samples were collected from the Khankra stream, from which 204 water mite species drifted. The least number of Hydrachnidia (694) were collected from Spot-1, and the highest (1809) were collected from Spot-2, with 106 water mites drifting from Spot-1 and 134 mites drifting from Spot-2 during the study period. From Spot-2 maximum 25 mite species were collected and minimum 19 mite species were collected from Spot-1. For various mite species, the DBDI value ranged from 0.155 (July) to 0.204 (April) in Spot-1 and 0.134 (July) to 0.149 (February) in Spot-2. It was also observed that maximum water mite species were day-drifter. The physico-chemical parameters of the Khankra stream were also reported. The effect of ecological parameters on mite drift was investigated using Canonical correspondence analysis (CCA).
\end{abstract}

Key Words: Water mites $\bullet$ Drift $\bullet$ Spring-fed stream $\bullet$ Water quality $\bullet$ Garhwal Himalaya $\bullet$ CCA.

\section{Introduction}

Drifting behavior of aquatic insects, including mites, have a great bearing on the ecosystem productivity and sustainability. The term "drift" was first of all coined by Muller (1954) to describe the downward movement of benthic invertebrates. According to Brittian and Eikeland (1988) the drifting is a natural mechanism in lotic ecosystems. Due to its significant role in the dispersal and colonization of aquatic organismsthe drift is considered a vital part of the lotic ecology (Towsend, 1989; Hughes, 1998).

Though the drift in benthic insects has been studied by several workers, yet the mites as such are largely ignored. Also, it is important to find out the ecological and interactive detrimental parameters which cause for insect drift. Certain environmental conditions make drifting species more likely to move away from the stream's bottom and be swept downstream. According to Schreiber (1995) in rivers and streams, invertebrate drift is a natural phenomenon that plays an important role in the ecological cycles. Waters (1962), Muller (1974), and Elliott (1967) were the pioneer to study invertebrate drift diel periodicity, and their results piqued the interest of a number of other researchers (Flecker, 1992; Brewin and Ormerod, 1994). Drift is an important consideration in the study of macrobenthic 
ecosystems since it is correlated with secondary development of aquatic environment, an important source of fish food, and an effective way for some species to colonies new areas (Waters, 1972; Williams \& Williams, 1993; Allan, 1995; Pringle and Ramrez, 1998).

The distribution and density of water mites in Garhwal Himalayan region has been recently attempted by some workers (Kumar and Dobriyal, 1992; Kumar et al., 2006, 2007; Pesic et al., 2007a, b; Pesic and Panesar, 2008; Pesic et al., 2012; Pesic et al., 2019a,b; Bahuguna et al., 2019; Pesic et al., 2020 a, b; Bahuguna and Negi, 2020 and Bahuguna et al., 2020), but the drift behavior remains un explored. Bahuguna et al., (2020) observed macrozoobenthos drifting behaviour in Kyunja gad from Garhwal Himalaya. Bahuguna and Dobriyal (2020) investigated the drift patterns of aquatic mites for the first time in Randi gad from the Garhwal Himalayas. The current research aims to investigate the drift in aquatic mites and ecology of Khankra gad, a spring-fed stream of Garhwal Himalaya.

\section{Material and Methods}

Study area: The study was conducted in Khankra gad, a third-order perennial spring-fed stream of the Alaknanda River, which is situated near Khankra village of Rudraprayag district $\left(30^{\circ} 17^{\prime} 15.1^{\prime \prime} \mathrm{N}\right.$ and $\left.78^{\circ} 58^{\prime} 41.4^{\prime \prime} \mathrm{E}\right)$ in Uttarakhand. (Fig. 1). It originates from the Bansoun peak in Garhwal Himalaya. Two sampling sites were selected, Spot-1 was located upstream at $30^{\circ} 14^{\prime} 37.12^{\prime \prime} \mathrm{N}$ and $78^{\circ} 55^{\prime} 06.00^{\prime \prime} \mathrm{E}$, and Spot-2 was located downstream at $30^{\circ} 14^{\prime} 45.76^{\prime \prime} \mathrm{N}$ and $78^{\circ} 54^{\prime} 55.84$ "E. The Khankra stream is used to deliver water for drinking and irrigation purposes nearby. The stream has a sandy and rocky bottom with mostly pine as riparian vegetation.

\section{Sample analysis}

From July 2018 to June 2020, monthly sampling was carried out. In the morning, water samples were taken and physicochemical parameters were determined, like Temperature $\left({ }^{\circ} \mathrm{C}\right)$, Velocity $\left(\mathrm{m} . \mathrm{sec}^{-1}\right), \mathrm{pH}$, were analyzed at the spot. For further analysis of other parameters such as
Dissolved Oxygen (mg. $\left.\mathrm{l}^{-1}\right)$, Total Alkalinity (mg.1 $\left.{ }^{1}\right)$, Total Hardness $\left(\mathrm{mg} \cdot \mathrm{l}^{-1}\right)$, water samples were bring in the laboratory. Water samples were analyzed in accordance with Welch (1948) and APHA (2012).

Drift experiments were carried out at two sampling spots located along the stream. The drifting sampling methods was followed as per Smock (1996) and Bahuguna and Dobriyal (2020). A drift net of diameter $1 \mathrm{~m}$ was fixed at a depth of $0.2 \mathrm{~m}$ for $24 \mathrm{hrs}$. The net was set for 4 hours at each spot, which was replaced by another net. Mite samples were preserved on the spot in Koenike's fluid and brought to laboratory for further investigations. A number of keys were used to identify the water mite species (Kumar et.al., 2007; Cook,1967, 1974; Prasad,1974; Gerecke, 2003; Pesic and Panesar, 2008). For drift density study, the Dobriyal-Bahuguna Drifting Index (DBDI) was used.

The formulae for DBDI index:

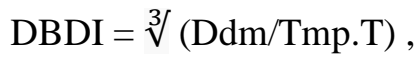

Where: D.B.D.I.= Dobriyal-Bahuguna Drifting Index.,

$\mathrm{Ddm}=$ No. of drifting mites (units per 0.04 $\mathrm{m}^{3} \cdot \mathrm{sec}^{-1}$ for 24 Hours), (i.e., mouth of net $1 \mathrm{~m}$, depth of sampler $0.2 \mathrm{~m}$, current velocity $0.2 \mathrm{~m} . \mathrm{sec}^{-}$ $\left.{ }^{1}\right)$, Tmp $=$ Total mite population in stream (units. $\mathrm{m}^{-2}$ )

$\mathrm{T}=$ Time used in collecting sample in 24 hours .

\section{Results}

Physico-chemical parameters: Table 1 displays the average (Mean \pm S.D) range of the physicochemical parameters of water collected at the two sampling sites of the stream. 


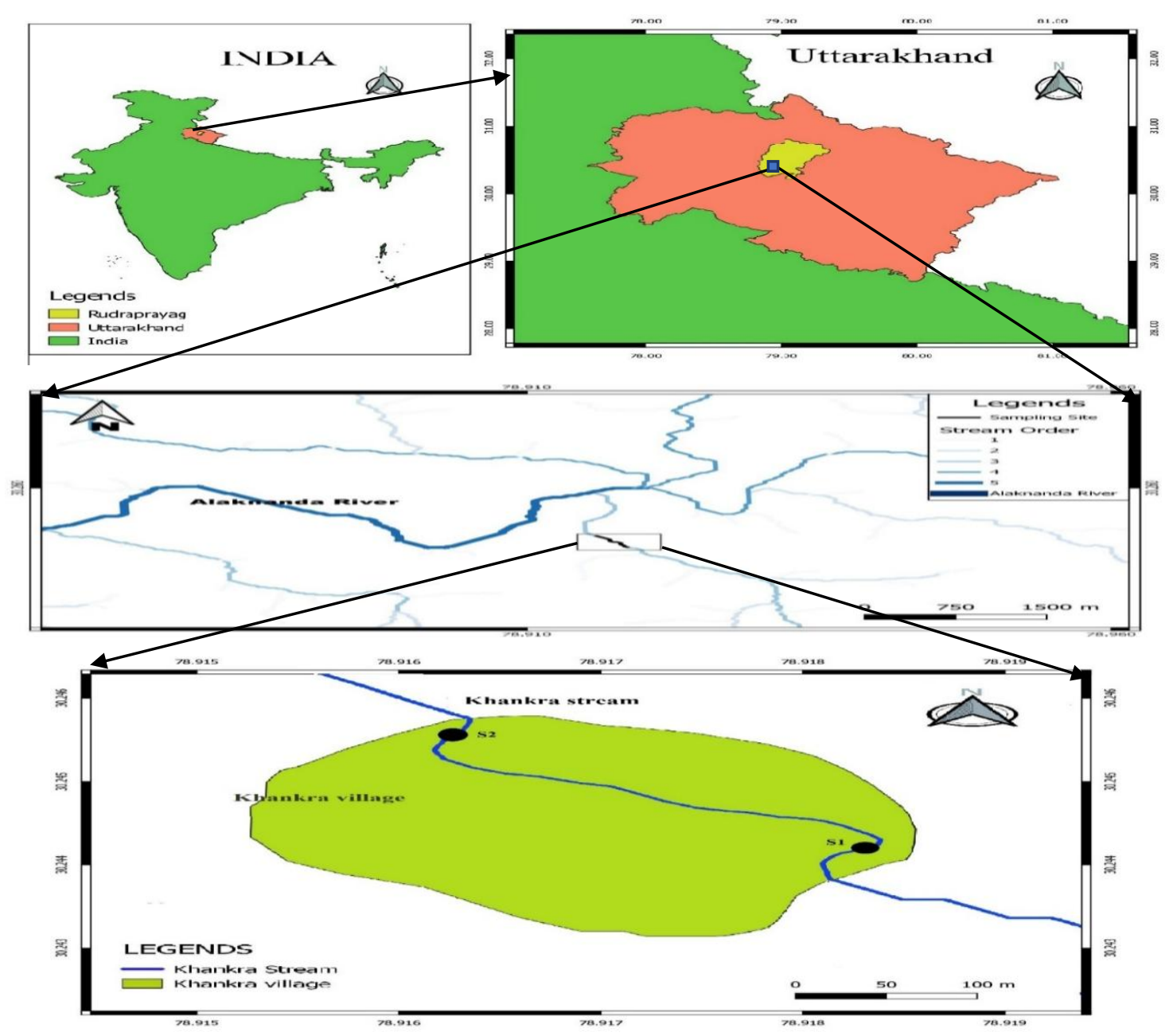

Fig 1. Sampling locations (Spot-1 and Spot-2) in Khankra gad, a spring-fed tributary of river Alaknanda, Uttarakhand, India.

Table 1: Average (Mean \pm S.D) range of the physico-chemical parameters of sampling Spots (Spot-1 and Spot-2) of Khankra gad stream during 2018-2020.

\begin{tabular}{lll}
\hline $\begin{array}{l}\text { Physico-chemical parameters } \\
(\mathbf{2 0 1 8 - 2 0})\end{array}$ & Spot-1 & Spot-2 \\
\hline WT $\left({ }^{\circ} \mathbf{C}\right)$ & $7.6 \pm 0.1$ to $12.8 \pm 0.2$ & $9.7 \pm 0.2$ to $18.4 \pm 0.8$ \\
pH & $7.4 \pm 0.1$ to $7.9 \pm 0.1$ & $7.3 \pm 0.1$ to $7.8 \pm 0.3$ \\
CV $\left(\mathbf{m . s e c}^{-\mathbf{1}}\right)$ & $0.26 \pm 0.02$ to $0.61 \pm 0.01$ & $0.25 \pm 0.01$ to $0.53 \pm 0.01$ \\
TA $\left(\mathbf{m g . l}^{-\mathbf{1}}\right)$ & $81.2 \pm 0.8$ to $99.1 \pm 1.1$ & $75.7 \pm 0.8$ to $94.7 \pm 1.3$ \\
TH $\left(\mathbf{m g . l}^{-1}\right.$ ) & $82 \pm 0.3$ to $121.9 \pm 6.2$ & $85.2 \pm 1.4$ to $109.8 \pm 13.2$ \\
DO $\left(\mathbf{m g . l}^{-1}\right.$ ) & $7.3 \pm 0.1$ to $8.8 \pm 0.3$ & $7.2 \pm 0.1$ to $8.4 \pm 0.1$ \\
\hline
\end{tabular}

The temperature of the water in the current study fairly good through-out the year, the values varied between $12.8 \pm 0.2$ to $7.6 \pm 0.1^{\circ} \mathrm{C}$ at Spot-1, and $9.7 \pm 0.2$ to $18.4 \pm 0.8{ }^{\circ} \mathrm{C}$ at Spot-2. The $\mathrm{pH}$ values ranged from $7.4 \pm 0.1$ to $7.9 \pm 0.1$ at Spot-1, and $7.3 \pm 0.1$ to $7.8 \pm 0.3$ at Spot-2. Velocity was recorded ranged between $0.61 \pm 0.01$ to $0.26 \pm 0.02$ $\mathrm{m} . \mathrm{sec}^{-1}$ at Spot-1, and $0.53 \pm 0.01$ to $0.25 \pm 0.01$ $\mathrm{m} \cdot \mathrm{sec}^{-1}$ at Spot-2. The total alkalinity observed was ranged between $81.2 \pm 0.8$ to $99.1 \pm 1.1 \mathrm{mg} . \mathrm{l}^{-1}$ at 
Spot-1, and $75.7 \pm 0.8$ to $94.7 \pm 1.3 \mathrm{mg} . \mathrm{l}^{-1}$ at Spot-2. The total hardness values were ranged from $82 \pm 0.3$ to $121.9 \pm 6.2 \mathrm{mg} . \mathrm{l}^{-1}$ at Spot- 1 , and $85.2 \pm 1.4$ to $109.8 \pm 13.2 \mathrm{mg} .1^{-1}$ at Spot-2. DO values ranged from $7.3 \pm 0.1$ to $8.8 \pm 0.3 \mathrm{mg} . \mathrm{l}^{-1}$ at Spot-1, and $7.2 \pm 0.1$ to $8.4 \pm 0.1 \mathrm{mg} . \mathrm{l}^{-1}$ at Spot-2 (Table. 1 ).

During the two-year study period, a total of 2503 mite samples were collected from Khankra stream, from which 204 water mite species drifted (Table 2 and Table 3).

\section{Dobriyal-Bahuguna Drifting Index (DBDI):}

DBDI index for Spot-1 and Spot-2 is shown in Table 4.. DBDI value ranged from 0.155 (July) to 0.204 (April) in Spot-1 and 0.134 (July) to 0.149 (February) in Spot-2. From DBDI values, it is revealed that April, May and August were conducive for mite population at Sot-1 and February and December at Spot-2.

\section{Diel drifting:}

Overall drifting pattern of water mites from Khankra stream is presented in the Fig.2 and Fig.3 respectively. Most of the drifting water mite species were caught during day time in Spot-1 (Fig. 2) and Spot-2 (Fig.3). At Spot-1 Torrenticola kumari, T. uttarakhandensis, Monatractides garhwaliensis, Sperchon indicus, S. garhwalensis, Atractides indicus, A. garhwali and Feltria

Total 25 mite species were collected from Spot-2, and a minimum of 19 mite species were collected from Spot-1. The highest diel drifting species were recorded in January and lowest in July month at both Spot-1 and spot-2. The least number of Hydrachnidia (694) were collected from Spot-1, and the highest (1809) were collected from Spot-2, with 106 water mites drifting from Spot-1 and 134 mites drifting from Spot-2.

In both sampling sites, the following species were observed: Torrenticola wonchoeli, $T$. uttarakhandensis, T. kumari, T. nana, T. semisuta, Monatractides oxystomus and M. garhwaliensis (family Torrenticolidae Piersig 1902), Sperchon garhwalensis, S. indicus (family Sperchontidae Thor, 1900), Atractides garhwali, A. incertus, A. indicus, A. ootacamundis and Hygrobates fluviatilis (Cook) (family Hygrobatidae). Torrenticola tetraporella, T. nana, Sperchonopsis verrucose, Atractides panesari, A. were daydrifter. Whereas, Torrenticola nana, T. muranyii, Monatractides kontschani, Sperchon ootacamundis, Atractides ootacamundis (Cook) were night-drifter and Torrenticola wonchoeli, $T$. semisuta, Monatractides oxystomus, Atractides incertus, Hygrobates fluviatilis, Feltria gereckei drifted in dawn time. At Spot-2 Torrenticola uttarakhandensis, T. chatterjeei, T. turkestanica, T. kumari, Monatractides garhwaliensis, Sperchon indicus, S. garhwalensis, Atractides indicus, A. garhwali and Hygrobates gangeticus were day-drifter. However, ootacamundis (Cook), Kongsbergia indica, and K. himalayaensis were night-drifter species and Torrenticola wonchoeli, Torrenticola semisuta, Monatractides Tuzovskyi, M. oxystomus, Atractides incertus, Hygrobates fluviatilis and Lebertia spp. drifted during dawn time. 
J. Mountain Res. P-ISSN: 0974-3030, E-ISSN: 2582-5011

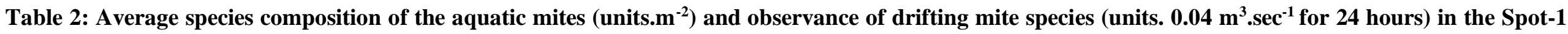
(S-1) of Khakhra stream of Garhwal, Uttarakhand (2018-20)

\begin{tabular}{|c|c|c|c|c|c|c|c|c|c|c|c|c|c|c|c|c|c|c|c|c|c|c|c|c|}
\hline \multirow[t]{2}{*}{ Name of Species } & \multicolumn{2}{|c|}{ Jul } & \multicolumn{2}{|c|}{ Aug } & \multicolumn{2}{|c|}{ Sep } & \multicolumn{2}{|c|}{ Oct } & \multicolumn{2}{|c|}{ Nov } & \multicolumn{2}{|c|}{ Dec } & \multicolumn{2}{|c|}{ Jan } & \multicolumn{2}{|c|}{ Feb } & \multicolumn{2}{|c|}{ Mar } & \multicolumn{2}{|c|}{ Apr } & \multicolumn{2}{|c|}{ May } & \multicolumn{2}{|c|}{ Jun } \\
\hline & $\mathbf{A}$ & B & $\mathbf{A}$ & B & A & B & $\mathbf{A}$ & B & $\mathbf{A}$ & B & $\mathbf{A}$ & B & A & B & $\mathbf{A}$ & B & $\mathbf{A}$ & B & $\mathbf{A}$ & B & A & B & $\overline{\mathbf{A}}$ & B \\
\hline \multirow{2}{*}{ Torrenticola uttarakhandensis } & 03 & $0 \pm 0$ & 02 & 01 & 05 & 01 & 04 & 01 & 10 & $02 \pm$ & $12 \pm$ & $02 \pm$ & $17 \pm$ & $03 \pm$ & $10 \pm$ & $01 \pm$ & $09 \pm$ & 02 & $07 \pm$ & $02 \pm$ & $04 \pm$ & $04 \pm$ & 01 & 01 \\
\hline & \pm 1.4 & & \pm 1.4 & \pm 1.4 & \pm 2.8 & \pm 1.4 & \pm 1.4 & \pm 1.4 & \pm 2.8 & 1.4 & 2.8 & 2.8 & 5.6 & 2.8 & 2.8 & 1.4 & 2.1 & \pm 1.4 & 1.4 & 1.4 & 2.8 & 2.8 & \pm 0 & \pm 0 \\
\hline \multirow[t]{2}{*}{ Torrenticola wonchoeli } & $0 \pm 0$ & $0 \pm 0$ & $0 \pm 0$ & $0 \pm 0$ & 02 & $0 \pm 0$ & $0 \pm 0$ & $0 \pm 0$ & $05 \pm$ & $0 \pm 0$ & $0 \pm 0$ & $0 \pm 0$ & $09 \pm$ & $01 \pm$ & $06 \pm$ & $01 \pm$ & $04 \pm$ & $01 \pm$ & $0 \pm 0$ & $0 \pm 0$ & $0 \pm 0$ & $0 \pm 0$ & 01 & $0 \pm 0$ \\
\hline & & & & & \pm 1.4 & & & & 1.4 & & & & 1.4 & 1.4 & 1.4 & 1.4 & 2.8 & 1.4 & & & & & \pm 1.4 & \\
\hline Torrenticola nana & $\begin{array}{c}02 \\
+1.4\end{array}$ & $0 \pm 0$ & $\begin{array}{c}01 \\
+1.4\end{array}$ & $0 \pm 0$ & $0 \pm 0$ & $0 \pm 0$ & $\begin{array}{c}04 \\
+2.8\end{array}$ & $01 \pm 1.4$ & $\begin{array}{l}01 \pm \\
1.4\end{array}$ & $0 \pm 0$ & $\begin{array}{l}04 \pm \\
2.8\end{array}$ & $\begin{array}{l}01 \pm \\
1.4\end{array}$ & $\begin{array}{l}09 \pm \\
2.8\end{array}$ & $\begin{array}{l}01 \pm \\
1.4\end{array}$ & $0 \pm 0$ & $0 \pm 0$ & $\begin{array}{l}02 \\
\pm 0\end{array}$ & $0 \pm 0$ & $0 \pm 0$ & $0 \pm 0$ & $0 \pm 0$ & $0 \pm 0$ & $0 \pm 0$ & $0 \pm 0$ \\
\hline \multirow{2}{*}{ Torrenticola kumari } & 01 & $0 \pm 0$ & $0 \pm 0$ & $0 \pm 0$ & 01 & $0 \pm 0$ & $0 \pm 0$ & $0 \pm 0$ & $02 \pm$ & $0 \pm 0$ & $06 \pm$ & $01 \pm$ & $07 \pm$ & $01 \pm$ & $04 \pm$ & $01 \pm$ & $0 \pm 0$ & $0 \pm 0$ & $03 \pm$ & $01 \pm$ & $05 \pm$ & $05 \pm$ & $03 \pm$ & $0 \pm 0$ \\
\hline & \pm 1.4 & & & & \pm 0 & & & & 1.4 & & 4.2 & 1.4 & 2.8 & 1.4 & 2.8 & 1.4 & & & 1.4 & 1.4 & 1.4 & 4.2 & 2.8 & \\
\hline \multirow[t]{2}{*}{ Torrenticola semisuta } & $0 \pm 0$ & $0 \pm 0$ & $0 \pm 0$ & $0 \pm 0$ & $0 \pm 0$ & $0 \pm 0$ & 01 & $0 \pm 0$ & $0 \pm 0$ & $0 \pm 0$ & $06 \pm$ & $01 \pm 0$ & $11 \pm$ & $01 \pm$ & $03 \pm$ & $01 \pm$ & $0 \pm 0$ & $0 \pm 0$ & $04 \pm$ & $0 \pm 0$ & $0 \pm 0$ & $0 \pm 0$ & $0 \pm 0$ & $0 \pm 0$ \\
\hline & & & & & & & \pm 0 & & & & 4.2 & & 2.8 & 1.4 & 1.4 & 1.4 & & & 2.8 & & & & & \\
\hline Torrenticola muranyii & $0 \pm 0$ & $0 \pm 0$ & $\begin{array}{c}02 \\
\pm 1.4\end{array}$ & $0 \pm 0$ & $0 \pm 0$ & $0 \pm 0$ & $\begin{array}{c}02 \\
\pm 1.4\end{array}$ & $0 \pm 0$ & $0 \pm 0$ & $0 \pm 0$ & $\begin{array}{l}04 \pm \\
2.8\end{array}$ & $01 \pm 0$ & $\begin{array}{l}08 \pm \\
2.8\end{array}$ & $0 \pm 0$ & $0 \pm 0$ & $0 \pm 0$ & $03 \pm 1.4$ & $0 \pm 0$ & $01 \pm 0$ & $0 \pm 0$ & $0 \pm 0$ & $0 \pm 0$ & $0 \pm 0$ & $0 \pm 0$ \\
\hline Monatractides garhwaliensis & 01 & $0 \pm 0$ & 03 & 01 & $0 \pm 0$ & $0 \pm 0$ & 04 & $0 \pm 0$ & $09 \pm$ & $01 \pm 1.4$ & $11 \pm$ & $02 \pm$ & $17 \pm$ & $03 \pm$ & $05 \pm$ & $01 \pm$ & $06 \pm$ & $0 \pm 0$ & $0 \pm 0$ & $0 \pm 0$ & $04 \pm 1.4$ & $04 \pm$ & $02 \pm$ & $0 \pm 0$ \\
\hline Monatractides oxystomus & 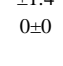 & $0 \pm 0$ & $0 \pm 0$ & $0 \pm 0$ & $\begin{array}{l}01 \\
\pm 0\end{array}$ & $0 \pm 0$ & $0 \pm 0$ & $0 \pm 0$ & $\begin{array}{l}04 \pm \\
1.4\end{array}$ & $0 \pm 0$ & $0 \pm 0$ & $0 \pm 0$ & $\begin{array}{l}07 \pm \\
2.8\end{array}$ & $0 \pm 0$ & $0 \pm 0$ & $\begin{array}{l}1.4 \\
0 \pm 0\end{array}$ & $\begin{array}{l}1.4 \\
0 \pm 0\end{array}$ & $0 \pm 0$ & $\begin{array}{l}03 \pm \\
1.4\end{array}$ & $\begin{array}{l}01 \pm \\
1.4\end{array}$ & $0 \pm 0$ & $\begin{array}{c}0 \\
0 \pm 0\end{array}$ & $\begin{array}{l}1.4 \\
0 \pm 0\end{array}$ & $0 \pm 0$ \\
\hline Monatractides kontschani & $0 \pm 0$ & $0 \pm 0$ & $\begin{array}{c}01 \\
+1.4\end{array}$ & $0 \pm 0$ & $0 \pm 0$ & $0 \pm 0$ & $\begin{array}{l}02 \\
\pm 0\end{array}$ & $\begin{array}{c}02 \\
+1.4\end{array}$ & $04 \pm$ & $01 \pm 1.4$ & $0 \pm 0$ & $0 \pm 0$ & $\begin{array}{c}05 \pm \\
8\end{array}$ & $0 \pm 0$ & $\begin{array}{l}03 \pm \\
1.4\end{array}$ & $\begin{array}{l}01 \pm \\
1.4\end{array}$ & $0 \pm 0$ & $0 \pm 0$ & $03 \pm$ & $01 \pm$ & $0 \pm 0$ & $0 \pm 0$ & $01 \pm 0$ & $0 \pm 0$ \\
\hline Sperchon indicus & 03 & 01 & $05 \pm$ & 01 & 07 & 02 & 09 & 01 & $11 \pm$ & $03 \pm 2.8$ & $14 \pm$ & $02 \pm$ & $21 \pm$ & $03 \pm$ & $16 \pm$ & $01 \pm$ & $11 \pm$ & 02 & $09 \pm$ & $02 \pm$ & $07 \pm 1.4$ & $07 \pm$ & $02 \pm$ & $0 \pm 0$ \\
\hline & \pm 1.4 & \pm 1.4 & 2.8 & \pm 1.4 & \pm 2.8 & \pm 1.4 & \pm 4.2 & \pm 1.4 & 2.8 & & 2.8 & 2.8 & 2.8 & 2.8 & 1.4 & 1.4 & 1.4 & \pm 1.4 & 2.8 & 1.4 & & 2.8 & 1.4 & \\
\hline Sperchon garhwalensis & $0 \pm 0$ & $0 \pm 0$ & $01 \pm$ & $0 \pm 0$ & $04 \pm$ & $01 \pm$ & $07 \pm$ & 0 & $09 \pm$ & $01 \pm 1.4$ & $11 \pm$ & $02 \pm$ & $15 \pm$ & $01 \pm$ & $09 \pm$ & $01 \pm$ & $08 \pm$ & $0 \pm 0$ & $06 \pm$ & $01 \pm$ & 02 & $02 \pm$ & $02 \pm$ & $0 \pm 0$ \\
\hline & & & 1.4 & & 1.4 & 1.4 & 4.2 & \pm 0 & 1.4 & & 1.4 & 1.4 & 5.6 & 1.4 & 1.4 & 1.4 & 1.4 & & 2.8 & 1.4 & \pm 0 & 1.4 & 2.8 & \\
\hline Sperchon ootacamundis & $0 \pm 0$ & $0 \pm 0$ & $0 \pm 0$ & $0 \pm 0$ & $0 \pm 0$ & $0 \pm 0$ & $0 \pm 0$ & $01 \pm 1.4$ & $01 \pm 1.4$ & $0 \pm 0$ & $02 \pm$ & $0 \pm 0$ & $08 \pm$ & $0 \pm 0$ & $0 \pm 0$ & $0 \pm 0$ & $01 \pm 0$ & $0 \pm 0$ & $0 \pm 0$ & $0 \pm 0$ & $0 \pm 0$ & $0 \pm 0$ & $01 \pm$ & $\begin{array}{l}01 \\
+0\end{array}$ \\
\hline Atractides indicus & 01 & $0 \pm 0$ & 05 & 01 & 04 & 01 & $07 \pm$ & $0 \pm 0$ & $09 \pm 7.1$ & $02 \pm 2.8$ & $\begin{array}{l}1.4 \\
12 \pm\end{array}$ & $01 \pm 1.4$ & $\begin{array}{l}2.8 \\
17 \pm\end{array}$ & $01 \pm$ & $09 \pm 2.8$ & $0 \pm 0$ & $04 \pm$ & 02 & $05 \pm$ & $0 \pm 0$ & $03 \pm$ & $03 \pm$ & $\begin{array}{l}1.4 \\
02 \pm\end{array}$ & $0 \pm 0$ \\
\hline & \pm 1.4 & & \pm 2.8 & \pm 1.4 & \pm 1.4 & \pm 1.4 & 4.2 & & & & 2.8 & & 7.1 & 1.4 & & & 2.8 & \pm 1.4 & 1.4 & & 1.4 & 1.4 & 2.8 & \\
\hline Atractides garhwali & $0 \pm 0$ & $0 \pm 0$ & $03 \pm$ & $0 \pm 0$ & $0 \pm 0$ & $0 \pm 0$ & $07 \pm$ & $01 \pm$ & $06 \pm$ & $0 \pm 0$ & $09 \pm$ & $0 \pm 0$ & $14 \pm$ & $01 \pm$ & $08 \pm$ & $0 \pm 0$ & $04 \pm$ & $0 \pm 0$ & $03 \pm$ & $01 \pm$ & $0 \pm 0$ & $0 \pm 0$ & $0 \pm 0$ & $0 \pm 0$ \\
\hline & & & 1.4 & & & & 1.4 & 1.4 & 1.4 & & 2.8 & & 2.8 & 1.4 & 1.4 & & 2.8 & & 1.4 & 1.4 & & & & \\
\hline Atractides incertus & $0 \pm 0$ & $0 \pm 0$ & $01 \pm$ & $0 \pm 0$ & $0 \pm 0$ & $0 \pm 0$ & $04 \pm$ & $0 \pm 0$ & $01 \pm 0$ & $0 \pm 0$ & $03 \pm$ & $01 \pm$ & $04 \pm$ & $01 \pm$ & $0 \pm 0$ & $0 \pm 0$ & $0 \pm 0$ & $0 \pm 0$ & $02 \pm$ & $0 \pm 0$ & $03 \pm$ & $03 \pm$ & $0 \pm 0$ & $0 \pm 0$ \\
\hline & & & 1.4 & & & & 2.8 & & & & 1.4 & 1.4 & 1.4 & 1.4 & & & & & 1.4 & & 1.4 & 2.8 & & \\
\hline Atractides ootacamundis (Cook) & $0 \pm 0$ & $0 \pm 0$ & $0 \pm 0$ & $0 \pm 0$ & 01 & $0 \pm 0$ & $02 \pm$ & $0 \pm 0$ & $0 \pm 0$ & $0 \pm 0$ & $0 \pm 0$ & $0 \pm 0$ & $01 \pm$ & $01 \pm$ & $0 \pm 0$ & $0 \pm 0$ & $03 \pm$ & $0 \pm 0$ & $0 \pm 0$ & $0 \pm 0$ & 01 & 01 & $0 \pm 0$ & $0 \pm 0$ \\
\hline & & & & & \pm 1.4 & & 1.4 & & & & & & 1.4 & 1.4 & & & 1.4 & & & & \pm 0 & \pm 0 & & \\
\hline Hygrobates fluviatilis & $0 \pm 0$ & $0 \pm 0$ & $0 \pm 0$ & $0 \pm 0$ & 03 & $0 \pm 0$ & $0 \pm 0$ & $0 \pm 0$ & 01 & 01 & 02 & $01 \pm$ & 03 & $01 \pm$ & $0 \pm 0$ & $0 \pm 0$ & 01 & 01 & $0 \pm 0$ & $0 \pm 0$ & 01 & 01 & 01 & $0 \pm 0$ \\
\hline & & & & & \pm 2.8 & & & & \pm 1.4 & \pm 0 & \pm 1.4 & 1.4 & \pm 0 & 1.4 & & & \pm 0 & \pm 0 & & & \pm 0 & \pm 0 & \pm 1.4 & \\
\hline Feltria gereckei & $0 \pm 0$ & $0 \pm 0$ & $0 \pm 0$ & $0 \pm 0$ & $0 \pm 0$ & $0 \pm 0$ & 01 & $0 \pm 0$ & $0 \pm 0$ & 01 & $0 \pm 0$ & $0 \pm 0$ & $02 \pm$ & $0 \pm 0$ & $01 \pm$ & $01 \pm$ & $0 \pm 0$ & $0 \pm 0$ & $03 \pm$ & $01 \pm$ & $0 \pm 0$ & $0 \pm 0$ & $01 \pm$ & $0 \pm 0$ \\
\hline & & & & & & & \pm 0 & & & \pm 0 & & & 1.4 & & 1.4 & 1.4 & & & 1.4 & 1.4 & & & 1.4 & \\
\hline Feltria indica & $0 \pm 0$ & $0 \pm 0$ & $0 \pm 0$ & $0 \pm 0$ & $02 \pm$ & $0 \pm 0$ & $01 \pm$ & $0 \pm 0$ & $0 \pm 0$ & $0 \pm 0$ & $0 \pm 0$ & $0 \pm 0$ & $02 \pm 0$ & $01 \pm$ & $01 \pm 0$ & $0 \pm 0$ & $0 \pm 0$ & $0 \pm 0$ & $0 \pm 0$ & $0 \pm 0$ & $0 \pm 0$ & $0 \pm 0$ & $0 \pm 0$ & $0 \pm 0$ \\
\hline TOTAL & $11 \pm$ & $01 \pm$ & & & $\begin{array}{l}1.42 \\
\mathbf{3 1 \pm}\end{array}$ & $05 \pm$ & $\begin{array}{l}1.4 \\
\mathbf{5 5 \pm}\end{array}$ & $09 \pm 8.4^{\mathrm{b}}$ & $73 \pm$ & $12 \pm$ & $96 \pm$ & $15 \pm$ & $177 \pm 56.5^{\mathrm{a}}$ & $\begin{array}{l}1.4 \\
\mathbf{2 2 \pm}\end{array}$ & $75 \pm$ & & $56 \pm$ & $09 \pm$ & $49 \pm$ & $10 \pm$ & $30 \pm$ & $05 \pm$ & $17 \pm$ & $02 \pm$ \\
\hline 101AL & $\begin{array}{l}11 \pm \\
8.4^{\mathrm{a}}\end{array}$ & $\begin{array}{l}0 \pm \\
1.4^{\mathrm{b}}\end{array}$ & $\begin{array}{l}24 \pm \\
18.2^{\mathrm{a}}\end{array}$ & $\begin{array}{l}5.6^{6} \\
0.4\end{array}$ & $15.5^{\mathrm{a}}$ & $5.6^{b}$ & $29.6^{\mathrm{a}}$ & & $28.3^{\mathrm{a}}$ & $9.8^{b}$ & $32.5^{\mathrm{a}}$ & $16.9^{b}$ & & $24^{b}$ & $19.7^{\mathrm{a}}$ & $12.7^{b}$ & $17.6^{a}$ & $7^{\mathrm{b}}$ & $19.7^{\mathrm{a}}$ & $11.1^{b}$ & $9.8^{a}$ & $12.7^{\mathrm{b}}$ & $16.9^{\mathrm{a}}$ & $1.4^{b}$ \\
\hline
\end{tabular}

$\left\{\mathrm{A}=\right.$ Density of mite species(unit. $\mathrm{m}^{-2}$ ), $\mathrm{B}=$ No. of drifting species (unit. $0.04 \mathrm{~m}^{3} \cdot \mathrm{sec}^{-1}$ for 24 hour), $\mathrm{a}=$ Total density 
Table 3: Average species composition of the aquatic mites (units.m ${ }^{-2}$ ) and observance of drifting mite species (units. $0.04 \mathrm{~m}^{3} . \mathrm{sec}^{-1}$ for 24 hours) in the Spot-2 (S-2) of Khakhra stream of Garhwal, Uttarakhand (2018-20)

\begin{tabular}{|c|c|c|c|c|c|c|c|c|c|c|c|c|c|c|c|c|c|c|c|c|c|c|c|c|}
\hline \multirow[t]{2}{*}{ Name of Species } & \multicolumn{2}{|c|}{ Jul } & \multicolumn{2}{|c|}{ Aug } & \multicolumn{2}{|c|}{ Sep } & \multicolumn{2}{|c|}{ Oct } & \multicolumn{2}{|c|}{ Nov } & \multicolumn{2}{|c|}{ Dec } & \multicolumn{2}{|c|}{ Jan } & \multicolumn{2}{|c|}{ Feb } & \multicolumn{2}{|c|}{ Mar } & \multicolumn{2}{|c|}{ Apr } & \multicolumn{2}{|c|}{ May } & \multicolumn{2}{|c|}{ Jun } \\
\hline & $\mathbf{A}$ & B & $\mathbf{A}$ & B & $\mathbf{A}$ & B & $\mathbf{A}$ & B & $\mathbf{A}$ & B & $\mathbf{A}$ & B & $\mathbf{A}$ & $\mathbf{B}$ & $\mathbf{A}$ & B & $\mathbf{A}$ & B & $\mathbf{A}$ & B & $\mathbf{A}$ & $\mathbf{B}$ & $\mathbf{A}$ & B \\
\hline Torrenticola & $01 \pm$ & $0 \pm 0$ & $02 \pm$ & $01 \pm$ & $04 \pm$ & $01 \pm$ & $0 \pm 0$ & $0 \pm 0$ & $11 \pm$ & $02 \pm$ & $13 \pm$ & $02 \pm$ & $15 \pm$ & $02 \pm$ & $13 \pm$ & $01 \pm$ & $11 \pm$ & $01 \pm$ & $14 \pm$ & $0 \pm 0$ & $12 \pm$ & $02 \pm$ & $09 \pm$ & $01 \pm$ \\
\hline uttarakhandensis & 1.4 & & 1.4 & 1.4 & 2.8 & 1.4 & & & 1.4 & 1.4 & 5.6 & 1.4 & 2.8 & 0 & 4.2 & 1.4 & 1.4 & 1.4 & 1.4 & & 1.4 & 0 & 2.8 & 0 \\
\hline \multirow[t]{2}{*}{ Torrenticola chatterjeei } & $0 \pm 0$ & $0 \pm 0$ & $01 \pm$ & $0 \pm 0$ & $06 \pm$ & $0 \pm 0$ & $05 \pm$ & $01 \pm$ & $0 \pm 0$ & $0 \pm 0$ & $08 \pm$ & $01 \pm$ & $10 \pm$ & $0 \pm 0$ & $05 \pm$ & $01 \pm$ & $0 \pm 0$ & $0 \pm 0$ & $0 \pm 0$ & $0 \pm 0$ & $0 \pm 0$ & $0 \pm 0$ & $07 \pm$ & $0 \pm 0$ \\
\hline & & & 1.4 & & 2.8 & & 1.4 & 0 & & & 4.2 & 1.4 & 2.8 & & 2.8 & 0 & & & & & & & 1.4 & \\
\hline Torrenticola turkestanica & $0 \pm 0$ & $0 \pm 0$ & $0 \pm 0$ & $0 \pm 0$ & $0 \pm 0$ & $0 \pm 0$ & $0 \pm 0$ & $0 \pm 0$ & $\begin{array}{l}05 \pm \\
2.8\end{array}$ & $0 \pm 0$ & $0 \pm 0$ & $0 \pm 0$ & $\begin{array}{l}07 \pm \\
2.8\end{array}$ & $\begin{array}{l}01 \pm \\
1.4\end{array}$ & $0 \pm 0$ & $0 \pm 0$ & $03 \pm$ & $0 \pm 0$ & $0 \pm 0$ & $0 \pm 0$ & $08 \pm$ & $0 \pm 0$ & $0 \pm 0$ & $0 \pm 0$ \\
\hline Torrenticola wonchoeli & $0 \pm 0$ & $0 \pm 0$ & $0 \pm 0$ & $0 \pm 0$ & $0 \pm 0$ & $0 \pm 0$ & $\begin{array}{l}04 \pm \\
2.8\end{array}$ & $0 \pm 0$ & $\begin{array}{l}2.0 \\
0 \pm 0\end{array}$ & $0 \pm 0$ & $0 \pm 0$ & $0 \pm 0$ & $\begin{array}{c}2.8 \\
01 \pm \\
0\end{array}$ & $\begin{array}{c}1.4 \\
01 \pm \\
1.4\end{array}$ & $\begin{array}{l}06 \pm \\
2.8\end{array}$ & $0 \pm 0$ & $\begin{array}{c}1.4 \\
03 \pm \\
0\end{array}$ & $0 \pm 0$ & $0 \pm 0$ & $0 \pm 0$ & $\begin{array}{c}5.6 \\
01 \pm \\
0\end{array}$ & $0 \pm 0$ & $0 \pm 0$ & $0 \pm 0$ \\
\hline Torrenticola tetraporella & $0 \pm 0$ & $0 \pm 0$ & $0 \pm 0$ & $0 \pm 0$ & $0 \pm 0$ & $0 \pm 0$ & $\begin{array}{c}05 \pm \\
0\end{array}$ & $0 \pm 0$ & $\begin{array}{r}03 \pm \\
1.4\end{array}$ & $0 \pm 0$ & $\begin{array}{l}04 \pm \\
2.8\end{array}$ & $\begin{array}{l}01 \pm \\
1.4\end{array}$ & $\begin{array}{c}08 \pm \\
2.8\end{array}$ & $\begin{array}{c}01 \pm \\
1.4\end{array}$ & $0 \pm 0$ & $0 \pm 0$ & $\begin{array}{c}02 \pm \\
2.8\end{array}$ & $0 \pm 0$ & $0 \pm 0$ & $0 \pm 0$ & $0 \pm 0$ & $0 \pm 0$ & $0 \pm 0$ & $0 \pm 0$ \\
\hline \multirow[t]{2}{*}{ Torrenticola semisuta } & $02 \pm$ & $0 \pm 0$ & $03 \pm$ & $0 \pm 0$ & $05 \pm$ & $0 \pm 0$ & $21 \pm$ & $01 \pm$ & $12 \pm$ & $01 \pm$ & $11 \pm$ & $01 \pm$ & $13 \pm$ & $01 \pm$ & $11 \pm$ & $0 \pm 0$ & $17 \pm$ & $0 \pm 0$ & $16 \pm$ & $01 \pm$ & $10 \pm$ & $0 \pm 0$ & $07 \pm$ & $0 \pm 0$ \\
\hline & 2.8 & & 2.8 & & 1.4 & & 2.8 & 0 & 2.8 & 0 & 1.4 & 1.4 & 4.2 & 1.4 & 1.4 & & 5.6 & & 4.2 & 0 & 2.8 & & 2.8 & \\
\hline Torrenticola nana & $0 \pm 0$ & $0 \pm 0$ & $0 \pm 0$ & $0 \pm 0$ & $05 \pm$ & $0 \pm 0$ & $06 \pm$ & $0 \pm 0$ & $12 \pm$ & $01 \pm$ & $08 \pm$ & $01 \pm$ & $09 \pm$ & $01 \pm$ & $07 \pm$ & $0 \pm 0$ & $05 \pm$ & $0 \pm 0$ & $07 \pm$ & $0 \pm 0$ & $04 \pm$ & $0 \pm 0$ & $0 \pm 0$ & $0 \pm 0$ \\
\hline & & & & & 2.8 & & 1.4 & & 4.2 & 1.4 & 1.4 & 1.4 & 1.4 & 1.4 & 2.8 & & 1.4 & & 4.2 & & 1.4 & & & \\
\hline Torrenticola kumari & $0 \pm 0$ & $0 \pm 0$ & $02 \pm$ & $0 \pm 0$ & $09 \pm$ & $0 \pm 0$ & $07 \pm$ & $0 \pm 0$ & $10 \pm$ & $0 \pm 0$ & $10 \pm$ & $01 \pm$ & $11 \pm$ & $01 \pm$ & $10 \pm$ & $01 \pm$ & $06 \pm$ & $01 \pm$ & $09 \pm$ & $01 \pm$ & $08 \pm$ & $01 \pm$ & $08 \pm$ & $01 \pm$ \\
\hline & & & 2.8 & & 1.4 & & 1.4 & & 1.4 & & 4.2 & 0 & 1.4 & 0 & 2.8 & 0 & 1.4 & 1.4 & 5.6 & 1.4 & 2.8 & 1.4 & 4.2 & 1.4 \\
\hline Monatractides & $0 \pm 0$ & $0 \pm 0$ & $02 \pm$ & $0 \pm 0$ & $11 \pm$ & $01 \pm$ & $10 \pm$ & $0 \pm 0$ & $15 \pm$ & $03 \pm$ & $15 \pm$ & $02 \pm$ & $18 \pm$ & $02 \pm$ & $15 \pm$ & $0 \pm 0$ & $12 \pm$ & $01 \pm$ & $10 \pm$ & $01 \pm$ & $09 \pm$ & $01 \pm$ & $11 \pm$ & $01 \pm$ \\
\hline garhwaliensis & & & 2.8 & & 1.4 & 1.4 & 1.4 & & 1.4 & 1.4 & 5.6 & 0 & 4.2 & 0 & 2.8 & & 1.4 & 0 & 4.2 & 1.4 & 2.8 & 1.4 & 1.4 & 0 \\
\hline Monatractides tuzovskyi & $0 \pm 0$ & $0 \pm 0$ & $0 \pm 0$ & $0 \pm 0$ & $09 \pm$ & $0 \pm 0$ & $08 \pm$ & $01 \pm$ & $09 \pm$ & $01 \pm$ & $14 \pm$ & $01 \pm$ & $13 \pm$ & $0 \pm 0$ & $16 \pm$ & $0 \pm 0$ & $15 \pm$ & $01 \pm$ & $12 \pm$ & $01 \pm$ & $10 \pm$ & $01 \pm$ & $08 \pm$ & $0 \pm 0$ \\
\hline & & & & & 4.2 & & 4.2 & 1.4 & 2.8 & 1.4 & 4.2 & 1.4 & 2.8 & & 1.4 & & 2.8 & 0 & 2.8 & 1.4 & 1.4 & 1.4 & 4.2 & \\
\hline Monatractides oxystomus & $0 \pm 0$ & $0 \pm 0$ & $01 \pm$ & $0 \pm 0$ & $05 \pm$ & $0 \pm 0$ & $0 \pm 0$ & $0 \pm 0$ & $0 \pm 0$ & $0 \pm 0$ & $0 \pm 0$ & $0 \pm 0$ & $01 \pm$ & $01 \pm$ & $04 \pm$ & $0 \pm 0$ & $0 \pm 0$ & $0 \pm 0$ & $06 \pm$ & $0 \pm 0$ & $0 \pm 0$ & $0 \pm 0$ & $0 \pm 0$ & $0 \pm 0$ \\
\hline & & & 1.4 & & 1.4 & & & & & & & & 1.4 & 1.4 & 2.8 & & & & 2.8 & & & & & \\
\hline Sperchon indicus & $03 \pm$ & $01 \pm$ & $05 \pm$ & $0 \pm 0$ & $08 \pm$ & 02 & $15 \pm$ & $02 \pm$ & $19 \pm$ & $03 \pm$ & $25 \pm$ & $03 \pm$ & $27 \pm$ & $03 \pm$ & $27 \pm$ & $03 \pm$ & $20 \pm$ & $02 \pm$ & $18 \pm$ & $02 \pm$ & $15 \pm$ & $02 \pm$ & $12 \pm$ & $01 \pm$ \\
\hline & 2.8 & 1.4 & 4.2 & & 2.8 & & 2.8 & 1.4 & 4.2 & 1.4 & 7.1 & 1.4 & 5.6 & 1.4 & 2.8 & 1.4 & 1.4 & 1.4 & 8.4 & 1.4 & 4.2 & 1.4 & 4.2 & 0 \\
\hline Sperchon garhwalensis & $02 \pm$ & $0 \pm 0$ & $0 \pm 0$ & $0 \pm 0$ & $0 \pm 0$ & $0 \pm 0$ & $12 \pm$ & $01 \pm$ & $16 \pm$ & $0 \pm 0$ & $21 \pm$ & $02 \pm$ & $28 \pm$ & $0 \pm 0$ & $26 \pm$ & $03 \pm$ & $21 \pm$ & $02 \pm$ & $20 \pm$ & $02 \pm$ & $16 \pm$ & $03 \pm$ & $12 \pm$ & $01 \pm$ \\
\hline & 2.8 & & & & & & 4.2 & 0 & 4.2 & & 4.2 & 1.4 & 4.2 & & 5.6 & 1.4 & 1.4 & 1.4 & 4.2 & 1.4 & 2.8 & 0 & 4.2 & 1.4 \\
\hline Sperchon plumifer & $03 \pm$ & $0 \pm 0$ & $04 \pm$ & $0 \pm 0$ & $06 \pm$ & $0 \pm 0$ & $07 \pm$ & $0 \pm 0$ & $06 \pm$ & $0 \pm 0$ & $08 \pm$ & $0 \pm 0$ & $09 \pm$ & $0 \pm 0$ & $0 \pm 0$ & $0 \pm 0$ & $18 \pm$ & $01 \pm$ & $12 \pm$ & $01 \pm$ & $10 \pm$ & $0 \pm 0$ & $08 \pm$ & $0 \pm 0$ \\
\hline & 2.8 & & 2.8 & & 2.8 & & 1.4 & & 4.2 & & 4.2 & & 2.8 & & & & 1.4 & 0 & 2.8 & 0 & 1.4 & & 4.2 & \\
\hline Sperchonopsis verrucosa & $0 \pm 0$ & $0 \pm 0$ & $0 \pm 0$ & $0 \pm 0$ & $0 \pm 0$ & $0 \pm 0$ & $06 \pm$ & $0 \pm 0$ & $08 \pm$ & $0 \pm 0$ & $05 \pm$ & $0 \pm 0$ & $01 \pm$ & $0 \pm 0$ & $05 \pm$ & $01 \pm$ & $0 \pm 0$ & $0 \pm 0$ & $0 \pm 0$ & $0 \pm 0$ & $0 \pm 0$ & $0 \pm 0$ & $0 \pm 0$ & $0 \pm 0$ \\
\hline & & & & & & & 2.8 & & 1.4 & & 2.8 & & 1.4 & & 2.8 & 0 & & & & & & & & \\
\hline Atractides indicus & $04 \pm$ & $0 \pm 0$ & $04 \pm$ & $01 \pm 0$ & $12 \pm$ & $02 \pm$ & $16 \pm$ & $01 \pm$ & $19 \pm$ & $01 \pm$ & $21 \pm$ & $01 \pm$ & $25 \pm$ & $02 \pm$ & $21 \pm$ & $02 \pm$ & $18 \pm$ & $0 \pm 0$ & $16 \pm$ & $01 \pm$ & $13 \pm$ & $0 \pm 0$ & $11 \pm$ & $01 \pm$ \\
\hline & 2.8 & & 2.8 & & 2.8 & 1.4 & 2.8 & 1.4 & 4.2 & 1.4 & 9.8 & 1.4 & 9.8 & 1.4 & 1.4 & 1.4 & 1.4 & & 4.2 & 0 & 1.4 & & 1.4 & 1.4 \\
\hline Atractides garhwali & $0 \pm 0$ & $0 \pm 0$ & $0 \pm 0$ & $0 \pm 0$ & $05 \pm$ & $0 \pm 0$ & $10 \pm$ & $01 \pm$ & $13 \pm$ & $01 \pm$ & $14 \pm$ & $01 \pm$ & $18 \pm$ & $01 \pm$ & $22 \pm$ & $02 \pm$ & $17 \pm$ & $01 \pm$ & $12 \pm$ & $01 \pm$ & $14 \pm$ & $0 \pm 0$ & $09 \pm$ & $01 \pm$ \\
\hline & & & & & 2.8 & & 1.4 & 1.4 & 2.8 & 1.4 & 1.4 & 1.4 & 1.4 & 0 & 5.6 & 1.4 & 4.2 & 1.4 & 1.4 & 0 & 2.8 & & 2.8 & 1.4 \\
\hline Atractides incertus & $0 \pm 0$ & $0 \pm 0$ & $01 \pm$ & $0 \pm 0$ & $02 \pm$ & $0 \pm 0$ & $06 \pm$ & $01 \pm$ & $06 \pm$ & $0 \pm 0$ & $07 \pm$ & $0 \pm 0$ & $02 \pm$ & $0 \pm 0$ & $09 \pm$ & $01 \pm$ & $08 \pm$ & $01 \pm$ & $0 \pm 0$ & $0 \pm 0$ & $0 \pm 0$ & $0 \pm 0$ & $01 \pm$ & $0 \pm 0$ \\
\hline & & & 1.4 & & 0 & & 2.8 & 1.4 & 2.8 & & 4.2 & & 0 & & 2.8 & 1.4 & 2.8 & 1.4 & & & & & 0 & \\
\hline Atractides panesari & $0 \pm 0$ & $0 \pm 0$ & $0 \pm 0$ & $0 \pm 0$ & $0 \pm 0$ & $0 \pm 0$ & $05 \pm$ & $0 \pm 0$ & $0 \pm 0$ & $0 \pm 0$ & $06 \pm$ & $0 \pm 0$ & $08 \pm$ & $01 \pm$ & $04 \pm$ & $01 \pm$ & $03 \pm$ & $0 \pm 0$ & $0 \pm 0$ & $0 \pm 0$ & $0 \pm 0$ & $0 \pm 0$ & $0 \pm 0$ & $0 \pm 0$ \\
\hline & & & & & & & 4.2 & & & & 4.2 & & 5.6 & 1.4 & 2.8 & 1.4 & 1.4 & & & & & & & \\
\hline $\begin{array}{l}\text { Atractides ootacamundis } \\
\text { (Cook) }\end{array}$ & $0 \pm 0$ & $0 \pm 0$ & $0 \pm 0$ & $0 \pm 0$ & $\begin{array}{c}01 \pm \\
1.4\end{array}$ & $0 \pm 0$ & $0 \pm 0$ & $0 \pm 0$ & $\begin{array}{c}06 \pm \\
1.4\end{array}$ & $0 \pm 0$ & $0 \pm 0$ & $0 \pm 0$ & $\begin{array}{l}05 \pm \\
4.2\end{array}$ & $\begin{array}{c}01 \pm \\
1.4\end{array}$ & $0 \pm 0$ & $0 \pm 0$ & $0 \pm 0$ & $0 \pm 0$ & $\begin{array}{l}05 \pm \\
2.8\end{array}$ & $\begin{array}{c}01 \pm \\
0\end{array}$ & $0 \pm 0$ & $0 \pm 0$ & $\begin{array}{c}01 \pm \\
0\end{array}$ & $0 \pm 0$ \\
\hline
\end{tabular}


J. Mountain Res. P-ISSN: 0974-3030, E-ISSN: 2582-5011

Vol. 16(1), (2021), 61-75

DOI: https://doi.org/10.51220/jmr.v16il.6

\begin{tabular}{|c|c|c|c|c|c|c|c|c|c|c|c|c|c|c|c|c|c|c|c|c|c|c|c|c|}
\hline Hygrobates gangeticus & $0 \pm 0$ & $0 \pm 0$ & $\begin{array}{c}01 \pm \\
0\end{array}$ & $0 \pm 0$ & $0 \pm 0$ & $0 \pm 0$ & $0 \pm 0$ & $0 \pm 0$ & $0 \pm 0$ & $0 \pm 0$ & $0 \pm 0$ & $\overline{0 \pm 0}$ & $\begin{array}{c}06 \pm \\
1.4\end{array}$ & $\begin{array}{c}01 \pm \\
1.4\end{array}$ & $0 \pm 0$ & $0 \pm 0$ & $\begin{array}{l}08 \pm \\
2.8\end{array}$ & $\begin{array}{l}01 \pm \\
1.4\end{array}$ & $0 \pm 0$ & $0 \pm 0$ & $0 \pm 0$ & $0 \pm 0$ & $0 \pm 0$ & $0 \pm 0$ \\
\hline Hygrobates fluviatilis & $0 \pm 0$ & $0 \pm 0$ & $0 \pm 0$ & $0 \pm 0$ & $\begin{array}{l}01 \pm \\
1.4\end{array}$ & 00 & $\begin{array}{c}05 \pm \\
1.4\end{array}$ & $\begin{array}{c}01 \pm \\
1.4\end{array}$ & $\begin{array}{c}06 \pm \\
1.4\end{array}$ & $0 \pm 0$ & $\begin{array}{l}10 \pm \\
2.8\end{array}$ & $0 \pm 0$ & $\begin{array}{l}12 \pm \\
5.6\end{array}$ & $0 \pm 0$ & $\begin{array}{l}10 \pm \\
2.8\end{array}$ & $0 \pm 0$ & $\begin{array}{c}08 \pm \\
2.8\end{array}$ & $\begin{array}{c}01 \pm \\
1.4\end{array}$ & $\begin{array}{c}05 \pm \\
2.8\end{array}$ & $0 \pm 0$ & $0 \pm 0$ & $0 \pm 0$ & $\begin{array}{c}04 \pm \\
1.4\end{array}$ & $0 \pm 0$ \\
\hline Kongsbergia indica & $\begin{array}{l}02 \pm \\
1.4\end{array}$ & $0 \pm 0$ & $0 \pm 0$ & $0 \pm 0$ & $0 \pm 0$ & $0 \pm 0$ & $\begin{array}{l}06 \pm \\
2.8\end{array}$ & $\begin{array}{l}01 \pm \\
1.4\end{array}$ & $0 \pm 0$ & $0 \pm 0$ & $\begin{array}{c}09 \pm \\
5.6\end{array}$ & $0 \pm 0$ & $\begin{array}{l}10 \pm \\
7.1\end{array}$ & $0 \pm 0$ & $\begin{array}{c}09 \pm \\
5.6\end{array}$ & $0 \pm 0$ & $\begin{array}{l}06 \pm \\
2.8\end{array}$ & $\begin{array}{c}01 \pm \\
1.4\end{array}$ & $\begin{array}{l}03 \pm \\
1.4\end{array}$ & $0 \pm 0$ & $\begin{array}{l}05 \pm \\
1.4\end{array}$ & $0 \pm 0$ & $0 \pm 0$ & $0 \pm 0$ \\
\hline $\begin{array}{l}\text { Kongsbergia } \\
\text { himalayaensis }\end{array}$ & $0 \pm 0$ & $0 \pm 0$ & $0 \pm 0$ & $0 \pm 0$ & $0 \pm 0$ & $0 \pm 0$ & $\begin{array}{c}06 \pm \\
2.8\end{array}$ & $\begin{array}{c}01 \pm \\
1.4\end{array}$ & $0 \pm 0$ & $0 \pm 0$ & $\begin{array}{c}06 \pm \\
1.4\end{array}$ & $0 \pm 0$ & $\begin{array}{c}09 \pm \\
5.6\end{array}$ & $\begin{array}{l}01 \pm \\
1.4\end{array}$ & $0 \pm 0$ & $0 \pm 0$ & $0 \pm 0$ & $0 \pm 0$ & $0 \pm 0$ & $0 \pm 0$ & $0 \pm 0$ & $0 \pm 0$ & $0 \pm 0$ & $\begin{array}{c}01 \pm \\
1.4\end{array}$ \\
\hline Lebertia spp. & $0 \pm 0$ & $0 \pm 0$ & $\begin{array}{c}01 \pm \\
0\end{array}$ & $0 \pm 0$ & $0 \pm 0$ & $0 \pm 0$ & $0 \pm 0$ & $0 \pm 0$ & $\begin{array}{c}05 \pm \\
1.4\end{array}$ & $0 \pm 0$ & $0 \pm 0$ & $0 \pm 0$ & $\begin{array}{c}08 \pm \\
2.8\end{array}$ & $0 \pm 0$ & $\begin{array}{c}06 \pm \\
1.4\end{array}$ & $\begin{array}{c}02 \pm \\
1.4\end{array}$ & $\begin{array}{l}07 \pm \\
1.4\end{array}$ & $0 \pm 0$ & $\begin{array}{l}04 \pm \\
2.8\end{array}$ & $0 \pm 0$ & $0 \pm 0$ & $0 \pm 0$ & $0 \pm 0$ & $0 \pm 0$ \\
\hline TOTAL & $\begin{array}{c}17 \pm \\
16.9^{\mathrm{a}}\end{array}$ & $\begin{array}{l}01 \pm \\
1.4^{b}\end{array}$ & $\begin{array}{c}27 \pm \\
29.6^{\mathrm{a}}\end{array}$ & $\begin{array}{l}02 \pm \\
1.4^{\mathrm{b}}\end{array}$ & $\begin{array}{c}89 \pm \\
31.1^{\mathrm{a}}\end{array}$ & $\begin{array}{l}06 \pm \\
4.2^{b}\end{array}$ & $\begin{array}{l}160 \pm \\
45.2^{\mathrm{a}}\end{array}$ & $\underset{\mathrm{b}}{12 \pm 11.3}$ & $\begin{array}{l}181 \pm \\
48.1^{\mathrm{a}}\end{array}$ & $\begin{array}{l}13 \pm \\
9.8^{\mathrm{b}}\end{array}$ & $\begin{array}{l}215 \pm \\
77.7^{\mathrm{a}}\end{array}$ & $\begin{array}{c}17 \pm \\
14.1^{b}\end{array}$ & $\begin{array}{l}274 \pm \\
84.8^{\mathrm{a}}\end{array}$ & $\begin{array}{r}21 \pm \\
22.2^{b}\end{array}$ & $\begin{array}{l}226 \pm \\
57.9^{\mathrm{a}}\end{array}$ & $\begin{array}{c}18 \pm \\
11.3^{b}\end{array}$ & $208 \pm 42.4^{\mathrm{a}}$ & $\begin{array}{c}14 \pm \\
12.7^{\mathrm{b}}\end{array}$ & $\begin{array}{l}169 \pm \\
56.5^{\mathrm{a}}\end{array}$ & $\begin{array}{l}12 \pm \\
7.1^{b}\end{array}$ & $135 \pm 32.5$ & $10 \pm 5.6^{\mathrm{b}}$ & $08 \pm 35.3^{\mathrm{a}}$ & $\begin{array}{l}08 \pm \\
7.1^{b}\end{array}$ \\
\hline
\end{tabular}

$\left\{\mathrm{A}=\right.$ Density of mite species(unit. $\left.\mathrm{m}^{-2}\right), \mathrm{B}=$ No. of drifting species (unit. $0.04 \mathrm{~m}^{3} \cdot \mathrm{sec}^{-1}$ for 24 hour $), \mathrm{a}=$ Total density $\left.\left(\mathrm{T}_{\mathrm{mp}}\right), \mathrm{b}=\mathrm{Total} \mathrm{drift}\left(\mathrm{D}_{\mathrm{dm}}\right)\right\}$ 
Canonical Correspondence Analysis (CCA), between water mites drift and environmental method was used to determine the relationship variables (Fig 4 and 5).

Table 4: Dobriyal-Bahuguna Drifting Index (DBDI) of mites in Khakhra stream for the period of July 2018 to June 2020.

\begin{tabular}{lll}
\hline Months (2018-20) & DBDI for Spot 1 & DBDI for Spot 2 \\
\hline July & 0.155 & 0.134 \\
August & 0.191 & 0.145 \\
September & 0.188 & 0.141 \\
October & 0.189 & 0.146 \\
November & 0.189 & 0.144 \\
December & 0.186 & 0.148 \\
January & 0.173 & 0.147 \\
February & 0.188 & 0.149 \\
March & 0.188 & 0.141 \\
April & 0.204 & 0.143 \\
May & 0.191 & 0.145 \\
June & 0.169 & 0.145 \\
\hline
\end{tabular}

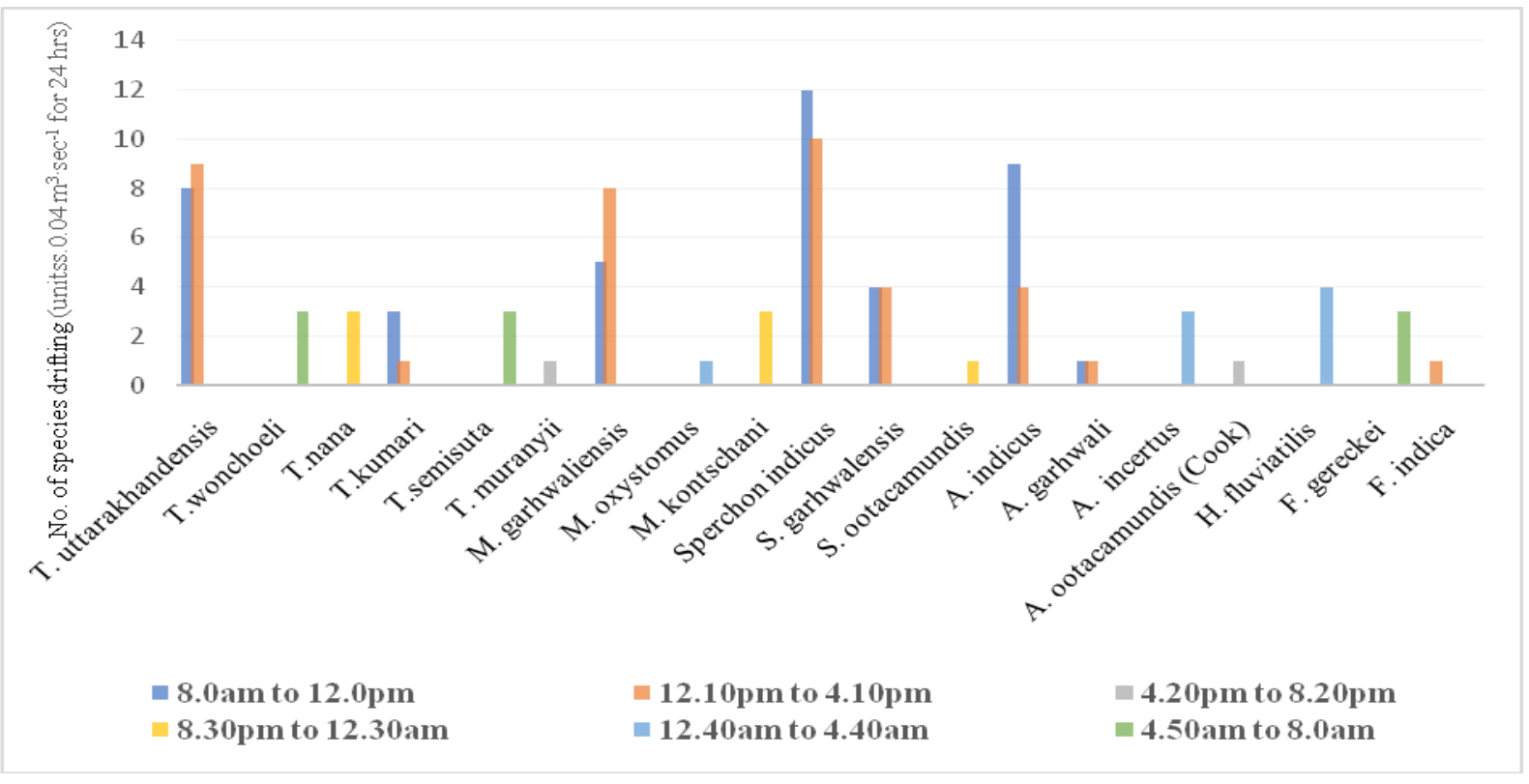

Fig. 2 Overall drifting pattern of aquatic mites in Spot-1 (S-1) of Khakhra stream (units. 0.04 $\mathrm{m}^{3} \cdot \mathrm{sec}^{-1}$ for 24 hours) for the period of July 2018 to June 2020. 


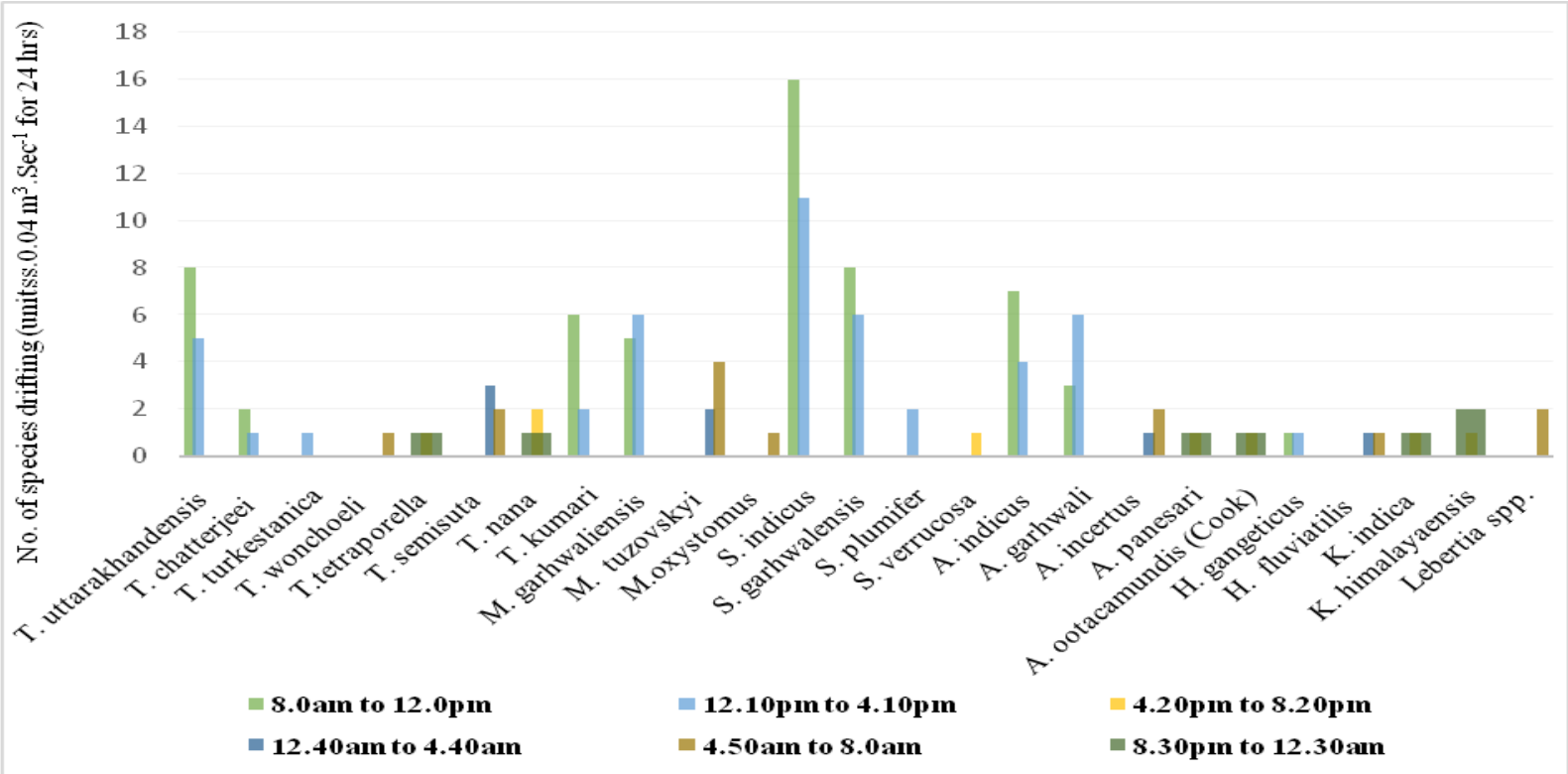

Fig. 3 Overall drifting pattern of aquatic mites in Spot-2 (S-2) of Khakhra stream (units. 0.04 $\mathrm{m}^{3} \cdot \mathrm{sec}^{-1}$ for 24 hours) for the period of July 2018 to June 2020.

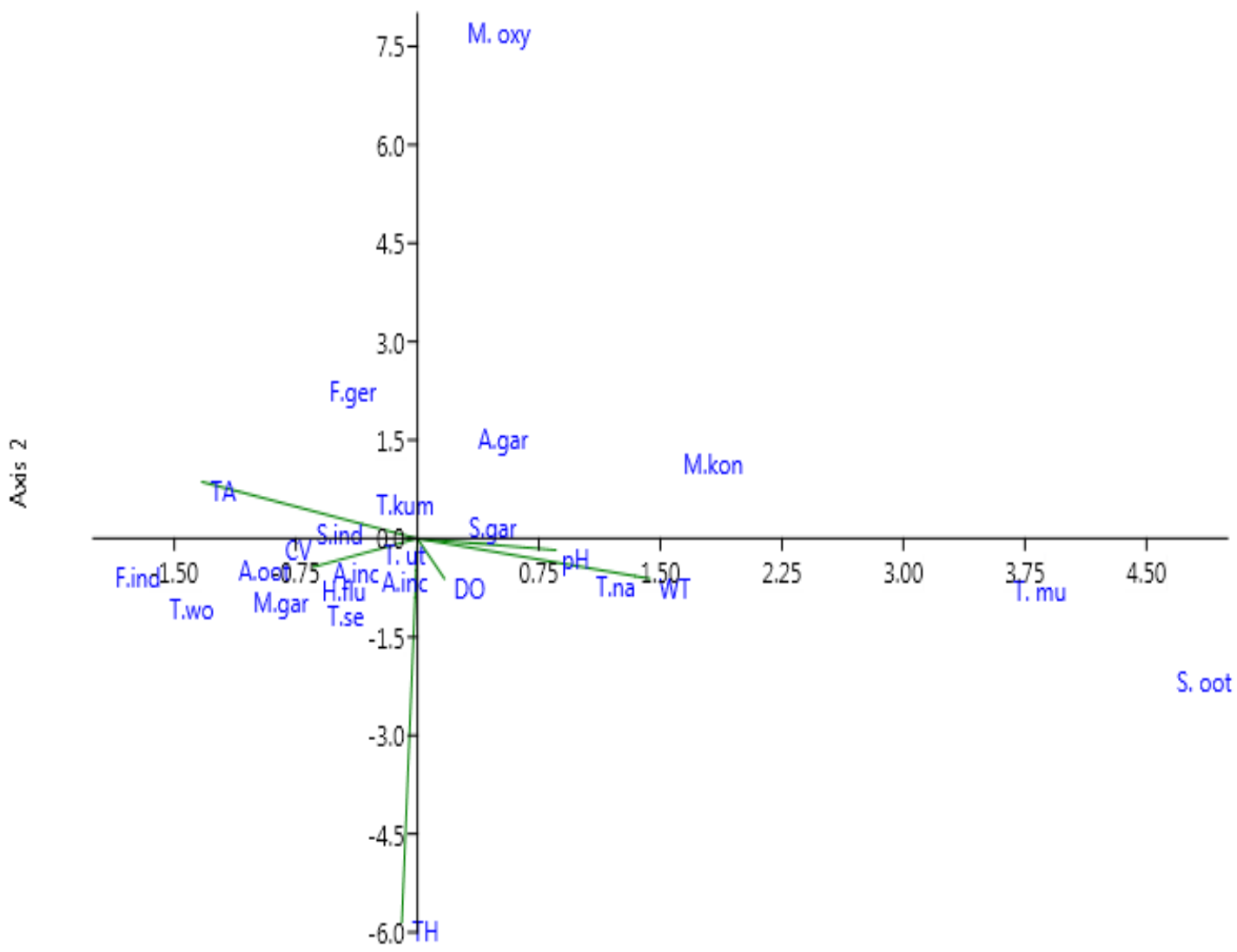

Axis 1

Fig.4 CCA bipot at Spot-1 between physico-chemical parameters and drifting water mite species during the period of 2018-20. 
(T. utt-T. uttarakhandensis, T. wo- $T$. wonchoeli, T. na- T. nana, T. kum-T. kumari, T. sem- T. semisuta, M. gar-M. garhwaliensis, M. oxy- M. oxystomus, S. ind- Sperchon indicus, S.gar- S. garhwalensis, T. mu- T. muranyii, M. kon- M. kontschani, F. gerFeltria gereckei, F. ind-F. indica, A.indAtractides indicus, A.gar- A. garhwali, A incA. incertus, A. oot-A. ootacamundis and H.flu-Hygrobates fluviatilis).

Each site's percentage of variance and Eigen values were found to be higher in axis 1 than in axis 2. Liu et al. (2010) observed similar findings. CCA was drawn between six physico- chemical parameters and 19 mite species at Spot1. At Spot-1, Eigen value for axis $1(0.244)$ explained $37 \%$ correlation and axis 2 (0.179) explained $27.88 \%$ correlation between physicochemical parameters and drifting species. DO, $\mathrm{pH}$ and water temperature indicate close relationship with T. nana, T muranyii, and S. garhwalensis. $S$. indicus, T. uttarakhandensis, a. incertus, himalayaensis, M. garhwaliensis, T. wonchoeli, F. indica, and $A$. ootacamundis (Cook) indicates a positive correlation with the current velocity. $M$. oxystomus was found to be least affected by these physico-chemical parameters.

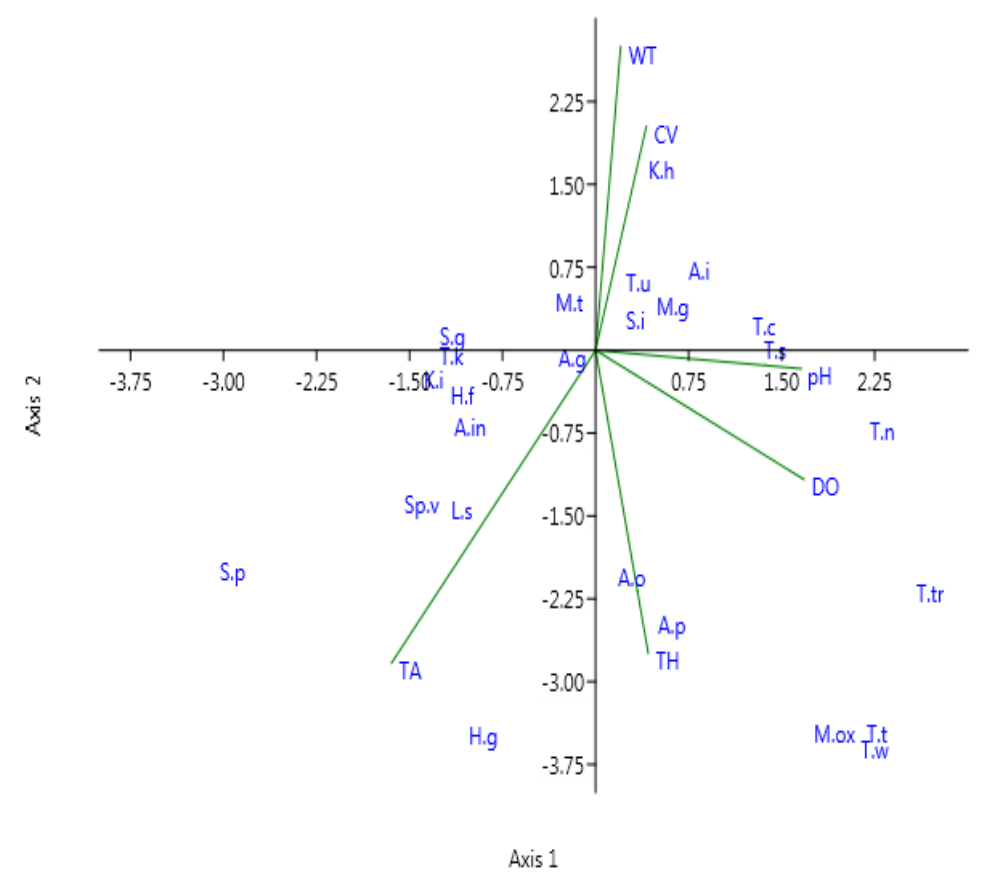

Fig.5 CCA bipot at Spot-2 between physico-chemical parameters and drifting water mite species during the period of 2018-20.

(T. utt-T. uttarakhandensis, T. wo- T. wonchoeli, T. na- T. nana, T. kum-T. kumari, T. sem- T. semisuta, M. gar-M. garhwaliensis, M. oxy- M. oxystomus, S. ind- Sperchon indicus, S.gar- S. garhwalensis, T. cha-T. chatterjeei, T. tur- $T$. turkestanica, T. tet- T. tetraporella, M. tuz- M. tuzovskyi, S.plu- S. plumifer, Sph. v-verrucosa, A. pan- A. panesari, H. gan- Hygrobates gangeticus, K. ind- Kongsbergia indica, K. himK. himalayaensis, L.spp-Lebertia spp., A.indAtractides indicus, A.garh- A. garhwali, A.inc-
A. incertus, T. oot- A. ootacamundis and H. fluHygrobates fluviatilis).

At Spot-2, 6 physico-chemical parameters and 25 species were taken into consideration to draw CCA (Fig. 3). At axis 1, Eigen value (0.167) explained $33.06 \%$ and Eigen value (0.124) value at axis 2 explained $24.53 \%$ correlation between physico-chemical parameters and drift mite species. T. nana, T. turkestanica, T. tetraporella, T. wonchoeli, M. oxystomus, and A. ootacamundis (Cook) indicate a positive relationship with DO, 
$\mathrm{pH}$, and total hardness. Sperchonopsis verrucosa, A. indicus, S. plumifer and A. garhwali show a positive correlation with total Alkalinity. $T$. uttarakhandensis, $K . \quad$ himalayaensis, $\quad T$. uttarakhandensis, A. incertus, M. garhwaliensis, T. chatterjeei, and T. semisuta show a positive correlation with water temperature and current velocity.

\section{Discussion}

To analyze stream ecology, certain physicochemical variables were examined. It was reported that water temperature values varied from $12.8 \pm 0.2$ to $7.6 \pm 0.1^{\circ} \mathrm{C}$ at Spot-1, and $9.7 \pm 0.2$ to $18.4 \pm 0.8{ }^{\circ} \mathrm{C}$ at Spot-2. Water temperature fluctuations disrupt the ecology of aquatic ecosystems. Chakrabarty et. al. (1959) discovered a similar trend in the Yamuna river. In the analysis, the $\mathrm{pH}$ of the water was mostly alkaline. The $\mathrm{pH}$ values ranged from $7.4 \pm 0.1$ to $7.9 \pm 0.1$ at Spot-1, and $7.3 \pm 0.1$ to $7.8 \pm 0.3$ at Spot-2. The $\mathrm{pH}$ of the water was mostly alkaline throughout the study period. The $\mathrm{pH}$ limits for drinking water set by the BIS (Bureau of Indian Standards) are 6.58.5.

The inclusion of limestone rocks increases the $\mathrm{pH}$ level (Ormerod et al., 1990). Strong primary production is aided by alkaline water (Kumar and Prabhahar, 2012). Throughout the year, velocity was reasonably consistent, with values ranging from $0.61 \pm 0.01$ to $0.26 \pm 0.02 \mathrm{~m} . \mathrm{sec}^{-1}$ at Spot-1, and $0.53 \pm 0.01$ to $0.25 \pm 0.01 \mathrm{~m} \cdot \mathrm{sec}^{-1}$ at Spot-2. Verma (2013) and Sharma et al. (2007) also reported a similar finding. Spot-1 DO values ranged from $7.3 \pm 0.1$ to $8.8 \pm 0.3 \mathrm{mg} . \mathrm{l}^{-1}$, while Spot2 DO values ranged from $7.2 \pm 0.1$ to $8.4 \pm 0.1 \mathrm{mg}^{-1}$ 1. Sharma et al. (2007) discovered a similar finding for DO in the Chandrabhaga stream of Garhwal Himalaya.

The amount of dissolved oxygen in natural and waste water is determined by the water body's physical, chemical, and biological activities. The WHO (World Health Organization) recommended that the DO norm be greater than $5.00 \mathrm{mg} / \mathrm{l}$. The presence of salt in the catchment area is expressed in the higher alkalinity levels. The overall alkalinity observed ranged from $81.2 \pm 0.8$ to $99.1 \pm 1.1 \mathrm{mg} . \mathrm{l}^{-1}$ at Spot-1, and from $75.7 \pm 0.8$ to $94.7 \pm 1.3 \mathrm{mg} . \mathrm{l}^{-1}$ at Spot-2. Because of the high concentrations of carbonates in natural water, it is predominantly alkaline (Todd, 1995). A similar finding was made by Kumar et al. (2010). The interaction of calcium and magnesium ions causes the water to be hard. Spot-1 total hardness values ranged from $82 \pm 0.3$ to $121.9 \pm 6.2 \mathrm{mg} . \mathrm{l}^{-1}$, while Spot-2 total hardness values ranged from $85.2 \pm 1.4$ to $109.8 \pm 13.2 \mathrm{mg} . \mathrm{l}^{-1}$. Researchers agree that biotic and abiotic parameters are inextricably linked, and that any change in one is likely to result in a significant change in the other. It has been noted that water temperature, $\mathrm{pH}$ and velocity are the main controlling factors, which can be directly correlated with density and drift behavior of aquatic insects (Bahuguna and Dobriyal, 2020, Dobriyal and Singh, 1981,1988, 1989). We may infer that the Khankra stream is not polluted and has good water quality based on the study of physicochemical parameters.

A major drift pattern of the water mites was observed in the current analysis, which varied in both sampling spots due to ecological factors. Nitrogen cycles, food chains, and invertebrate population patterns are all influenced by invertebrate drift, which is a significant ecological aspect of river systems. In the present study of Khankra gad spring-fed stream, the aquatic mites were recorded maximum in January and minimum in July in both the sampling spots. Many aquatic invertebrates exhibit diurnal periodicity in activity. According to Elliott (2002), the process of invertebrate drift often results in a rise in upstream migration. Some stream invertebrates become more active at night. In general, the density of drifting species is greatest at night and then at dusk. (Waters 1972 and Allan 1995). Water (1972) and Muller (1974) discovered the role of sunlight in the regulation of drift behaviour. Elliott (2002) and Bahuguna et al., (2020) observed how the distribution of benthic invertebrates in a rocky stream shifted during the day and night. Bahuguna et al. (2019) have discovered a connection between fish predation and Odonata drifting. 
The DBDI Index was used, which indicates the maximum number of mites that migrated away from the total number of mites present at the time and place (Bahuguna and Dobriyal, 2020). For drifting mites in Khankra gad, the DBDI value indicated monthly variations. DBDI value was higher in April (0.204) at Spot-1 and February (0.149) at Spot-2. From DBDI values reveled that April, May and August were conducive for mite population at Spot-1 and February and December at Spot-2. Most of the species were day-drifter. From Spot-1 maximum eight species i.e., Torrenticola uttarakhandensis, T. kumari, Monatractides garhwaliensis, Sperchon indicus, S. garhwalensis, Atractides indicus, A. garhwali and Feltria and from Spot-2 ten species i.e., Torrenticola uttarakhandensis, T. chatterjeei, T. turkestanica, T. kumari, Monatractides garhwaliensis, Sperchon indicus, S. garhwalensis, Atractides indicus, A. garhwali and Hygrobates gangeticus were found to be drifting at day time. Bahuguna and Dobriyal (2020) also reported that there was a clear preference for drifting during the day instead of drifting at evening or in the night. The drifting of aquatic insects in lotic water rises during the day, according to many reports using direct observations (Graesser and Lake 1984; Allan et.al. 1986).

Most stream invertebrates speed up their drifting behavior at night. In spot-1 five species i.e., Torrenticola nana, T. muranyii, Monatractides kontschani, Sperchon ootacamundis, Atractides ootacamundis (Cook) were night-drifter and from Spot-2 seven species i.e., Torrenticola tetraporella, T. nana, Sperchonopsis verrucose, Atractides panesari, A. ootacamundis (Cook), Kongsbergia indica, and $K$. himalayaensis were night-drifter. The drifting of invertebrates became more frequent at night (Bishop and Hynes, 1969). Dawn time drifting was also observed in the water mites of both the spots (Spot-1 and Spot-2) of Khankra stream. At Spot-1 six species i.e., Torrenticola wonchoeli, $T$. semisuta, Monatractides oxystomus, Atractides incertus, Hygrobates fluviatilis, Feltria gereckei and seven species i.e., Torrenticola wonchoeli, Torrenticola semisuta, Monatractides Tuzovskyi,
M. oxystomus, Atractides incertus, Hygrobates fluviatilis and Lebertia spp. from Spot-2 drifted during dawn time. Waters (1972) and Allan (1995) both observed aquatic insects drifting at dawn. There are a variety of hypotheses as to why these invertebrates drift. Drifts may be caused by a number of reasons, including the scramble for food and substratum, as well as predator avoidance and unfavourable environmental conditions (Hauer and Resh 2006).

CCA plot suggested that the few species were correlated with water temperature, $\mathrm{pH}, \mathrm{DO}, \mathrm{TH}$, and Total alkalinity in both spots, but the majority of the species were influenced by water velocity. Bahuguna et al., (2019); Bahuguna and Dobriyal, 2020 reported similar findings.

Based on the results of this analysis, it is possible to infer that some species displayed a specific diel drifting pattern. Drought and flood susceptibility, food, organism competition, shade, and zoogeography, as well as water current, temperature, substratum, vegetation, and dissolved substances, each of these factors influence the occurrence and distribution of stream invertebrates (Hynes 1970).

\section{Conclusion}

Current research summarizes the monthly variations of physicochemical parameters and their influence on the drifting behaviour of water mites in Khankra stream with an explanation. Drift is an essential characteristic of water mite species. Water mites drifting behaviour has an effect on the survival of other aquatic species. Upper stretch of the stream shows fewer drifting species, whereas in lower stretch more drifting species were recorded. Based on a number of conditions, it can be nocturnal, diurnal, or dawntime. Water mites drifting behaviour has an effect on the survival of other aquatic species.

\section{Acknowledgements}

The authors (SN and PB) are grateful for the financial assistance provided by the Science and Engineering Research Board (SERB) as a major project F. No. -ECR/2016/001291. 


\section{References}

Allan JD (1995). Stream ecology. Structure and function of running waters. Chapman \& Hall London, 1-388.

Allan JD Flecker A.S. and McClintock N.L. (1986). Diel epibenthic activity of mayfly nymphs and it nonconcordance with behavioural drift. Limnol. Oceanogr.31: 1057-1065.

APHA, (2012). Standard methods for the examination of water and waste water. APHA, AWWA, WPCF, 22th edition, New York

Bahuguna P, Joshi HK and Koshal K (2019) A report on drifting behaviour of odonata (aquatic insects) in Kyunja Gad, a spring fed tributary of river Mandakani, Chamoli Garhwal, Uttarakhand. J. Mountain Res. 14(2): 63-67.

Bahuguna, P., Negi, S. (2021). A checklist of the water mites (Acari: Hydrachnidia) of Garhwal Himalaya with some new records. (Edited by R. A. Singh, Conference Book on Geology and Natural Resource of Himalaya. A.S.R. Publication, Lucknow -226008.

Bahuguna, P., Negi, S., and Dobriyal, A. K. (2019). Density and diversity of aquatic mites in a spring fed stream of Garhwal Himalaya, India. J. Mountain Res., 14(2), 55-59.

Bahuguna, P., Rana, K. K., Rayal, R., and Joshi, H. K. (2020). Studies on the drifting behavioural patterns of macrozoobenthos in Kyunja Gad, a mountain stream of Garhwal himalya, india. J. Mountain. Res., (15), 97108.

Bahuguna, P., Rana, K. K., Rayal, R., and Khanduri, N. C. (2020). Density and diversity of aquatic mites in a glacier-fed river Mandakani from Garhwal Central Himalaya, India. Uttar Pradesh Journal of Zoology, 41(10): 1-8.

Bahuguna, Pankaj and Dobriyal, A. K. (2020). Population structure and drifting pattern of aquatic mites in Randi Gad, a tributary of River Alaknanda in Garhwal Himalaya, Uttarakhand, India. J. Mountain. Res., (15), 63-70.
Bishop JE and Hynes HBN (1969). Downstream drift of the invertebrate fauna in a stream ecosystem. Arch. Hydrobiol. 66:56-90.

Brewin P.A. \& Ormerod S.J. (1994) Macroinvertebrate drift in streams of the Nepalese Himalaya. Freshwat Biol., 32, 573 583.

Brittain, J.E. \& Eikeland, TJ. (1988). Invertebrate drift: a review. Hydrobiolgjia, 166: 77-9

Chakrabarty, R.D., Roy, P. and Singh, S.B. (1959). A quantative study of plankton and physicochemical conditions of river Yamuna at Allahabad. Indian J. Fish, 61:186-208.

Cook, D. R. (1967). Water mites from India. Memoirs of the American Entomological Institute, 9: 1-411.

Cook, D. R. and R. D. Mitchell (1953). Notes on collecting water-mites. Turtox News, 30,122125.

Dobriyal, A.K. and Kumar, N. (1988). Fish and fisheries of the river Mandakini. In: Khulbe, R.D. (Ed). Prospectives in aquatic biology. Papyrus publishing house. New Delhi. pp. 337-340.

Dobriyal, A.K. and Singh, H.R. (1981). Diurnal variation in some aspects of limnology of the river Mandakini from the Garhwal Himalaya U.P. Uttar Pradesh. J. Zool. 1: 16-18.

Dobriyal, A.K. and Singh, H.R. 1989. Observations on temporal trends of phytoplankton diversity in the river Nayar of Garhwal Himalaya. J. Freshwat Biol. 1: 1-6.

Elliott, J. (1973). The Diel Activity Pattern, Drifting and Food of the Leech Erpobdella octoculata (L.) (Hirudinea: Erpobdellidae) in a Lake District Stream. J Ani. Eco., 42(2), 449-459.

Elliott JM (2002) Time spent in the drift by downstream-dispersing invertebrates in a Lake District Stream. J. Freshwater Biol. 71: 112-122.

Hauer, F. R., \& Resh, V. H. (2007). Macroinvertebrates. In F. R. Hauer \& G. A. Lamberti (Eds.), Methods in Stream Ecology. (2nd edn., pp. 435-463). Elsevier Academic Press.

Hauer, F.R. \& V.H. Resh. (2006). Macroinvertebrates. In R.F. Hauer \& G.A. 
Lamberti (eds.), Methods in stream ecology ( $2^{\text {nd }}$ ed., pp. 435-463). Elsevier Academic Press, New York,

Flecker, A. S. (1992). Fish predation and the evolution of invertebrate drift periodicity: evidence from neotropical streams. Ecol. 73, 438-48.

Gerecke R. (2003). Water mites of the genus Atractides Koch, 1837 (Acari: Parasitengona: Hygrobatidae) in the western Palaearctic region: a review. Zoo. J. Linnean Soc., 138, 141-378.

Graesse A and Lake PS (1984). Diel changes in the benthos of stones and of drift in a Southern Australian upland stream. Hydrobiolo. 111:153-160.

Hughes N.F. (1998). A model of habitat selection by drift-feeding stream salmonids at different scales. Eco., 79(1): 281-294.

Hynes, H. B. N. (1970). The ecology of stream insects. Annu. Rev. Entomol.,15: 2542.

Kumar MP, Prabhahar C (2012). Physicochemical parameters of river water: a review. Int J Pharm Biol Arch, 3:1304-1312.

Kumar N and Dobriyal AK (1992). Some observations on the water mites of a Hill stream Khanda gad in Garhwal Himalaya. $J$. Freshwater Biol. 4: 193-197.

Kumar, A., Bisht, B.S., Talwar, A. and Chandel, D. (2010). Physico-chemical and microbial analysis of groundwater from different regions of Doon Valley. Int. J. Appl. Env. Sci, 5(3): 433-440.

Kumar, N., Kumar, K., Kumar, S. and Pesic, V. (2006). Monatractides tuzovskyi sp. nov. (Acari: Torrenticolidae), a new water mite species from the Garhwal Himalayas (India). Acarina, 14(2), 81-83.

Kumar, N., Kumar. K. and Pesic, V. (2007). Two new species of Sperchon Kramer (Acari: Hydrachnidia: Sperchontidae) from the Garhwal Himalayas (India). Syst. Appl. Acar 12, 31-36.

Liu, C., Liu, L., Shen, H., 2010. Seasonal variations of phytoplankton community structure in relation to physico-chemical factors in Lake Baiyangdian, China. Procedia Environ. Sci. 2, 1622-1631.

Muller K. (1954). Investigations on the organic drift in North Swedish streams. Rep. Inst. Freshw. Res. Drottningholm, 35: 133-148.

Muller K. (1974). Stream drift as a chronobiological phenomenon in running water ecosystems. Annu Rev Ecol Systemat 5: 309-323.

Ormerod, S. J., Weatherley, N. S., Merrett, W. J., Gee, A. S., \& Whitehead, P. G. (1990). Restoring acidified streams in upland Wales: A modelling comparison of the chemical and biological effects of liming and reduced sulphate deposition. Env. Poll., 64(1), 67-85.

Pesic, V. and Panesar, A. (2008). Studies on water mites (Acari, Hydrachnidia) from the Himalayas, I. The water mite genus Feltria Koenike, with descriptions of eight new species. Zootaxa, 1758, 1-28.

Pesic, V., Chatterjee, T., Das, M. K. and Bordoloi, S. (2012). Two rare water mite species (Acari, Hydrachnidia) from the streams of the Indian eastern Himalayan region. Syst. Appl. Acar., 17(4): 458-464.

Pesic, V., Kumar, N. and Kumar, K. (2007a). Two new species of water mites of the family Hygrobatidae (Acari: Hydrachnidia) from the Garhwal Himalayas (India). Syst. Appl. Acar. 12, 161-166.

Pesic, V., Kumar, N. and Kumar, K. (2007b). A new species of Monatractides (Acari: Hydrachnidia: Torrenticolidae) and new records of other torrenticolid water mites from the Garhwal Himalayas (India). Syst. Appl. Acar., 12(3-4),

Pesic, V., Smit, H. and Bahuguna, P. (2019a). New records of water mites (Acari: Hydrachnidia) from the West-ern Himalaya with the description of four new species. Syst. Appl. Acar., 24(1), 5980.

Pesic, V., Smit, H. and Bahuguna, P. (2019b). New records of water mites (Acari: Hydrachnidia) from the West-ern Himalaya and description of three new species from 
Asia. Syst. Appl. Acar., 24(10),1868- 1880. 20.

Pesic, V., Smit, H. and Bahuguna, P. (2020a). A new species of Kongsbergia from the Western Himalaya with a key to the species of the genus of India (Acari: Hydrachnidia). Ecol Monte, 27, 35-38. 21.

Pesic, V., Smit, H. and Bahuguna, P., Negi, S., Dobriyal, A. K. (2020b). Torrenticolid water mites of India with description of three new species (Acari: Hydrachnidia, Torrenticolidae), Syst. Appl. Acaro.,25(2), 255-267.

Prasad, (1974). A Catalogue of mites of India. Ludhiana, Indira Acarology Publishing House, 320 pp.

Pringle C.M. \& Ramı'rez A. (1998). Use of both benthic and drift sampling techniques to assess tropical stream invertebrate communities along an altitudinal gradient, Costa Rica. Freshwat. Biol., 39, 359-373.

Schreiber, E. S. G. (1995). Long-term patterns of invertebrate stream drift in an Australian temperate stream. Freshwat. Biol., 33, 13-25.

Sharma, A., Sharma R.C. and Anthwal, A. (2007). Monitoring phytoplankton diversity in the hill stream Chandrabhaga in Garhwal Himalayas. Life Sci. J., 4:80-84

Todd LP (1995). Changes in biological characteristics of Daphnia magna from chronic action of copper and nickel at low concentrations. Hydrobiol J, 5:59-62

Townsend C.R. (1989). The patch dynamics concept of stream community ecology. J. N. Am. Benthol. Soc., 8(1): 36-50.

Waters, T. F. (1962). Diurnal Periodicity in the Drift of Stream Invertebrates. Ecology, 43(2), 316-320.

Waters, T. F. (1972). The drift of stream insects. A. Rev. En. 17: 253-272.

Welch, P.S (1948). Limnological Methods. Blackiston Co. Philadephia.

Williams D.D. \& Williams N.E. (1993). The upstream /downstream movement paradox of lotic invertebrates: quantitative evidence from a Welsh mountain stream. Freshwat. Biol., 30, 199-218. 